\title{
Switching spatial scale reveals dominance-dependent social foraging tactics in a wild primate
}

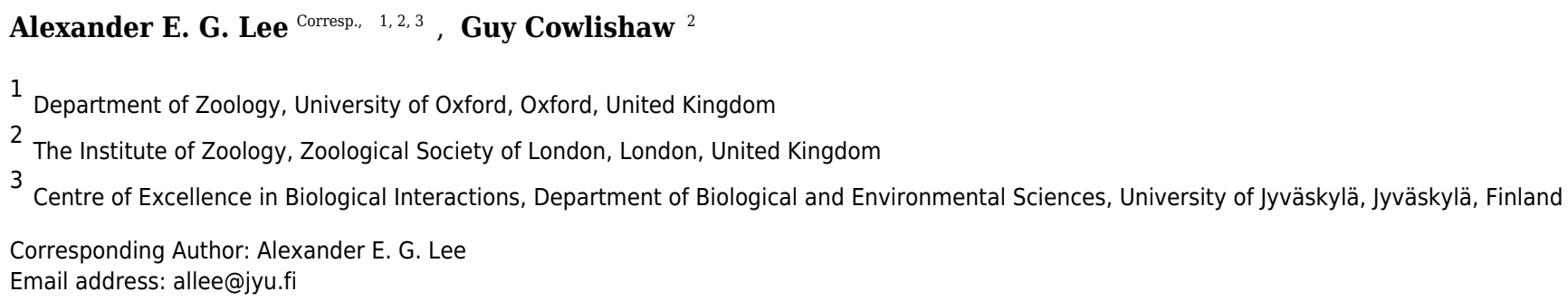

When foraging in a social group, individuals are faced with the choice of sampling their environment directly or exploiting the discoveries of others. The evolutionary dynamics of this trade-off have been explored mathematically through the producer-scrounger game, which has highlighted socially exploitative behaviours as a major potential cost of group living. However, our understanding of the tight interplay that can exist between social dominance and scrounging behaviour is limited. To date, only two theoretical studies have explored this relationship systematically, demonstrating that because scrounging requires joining a competitor at a resource, it should become exclusive to high-ranking individuals when resources are monopolisable. In this study, we explore the predictions of this model through observations of the natural social foraging behaviour of a wild population of chacma baboons (Papio ursinus). We collected data through over 800 hours of focal follows of 101 adults and juveniles across two troops over two 3-month periods. By recording over 7,900 social foraging decisions at two spatial scales we show that, when resources are large and economically indefensible, the joining behaviour required for scrounging can occur across all social ranks. When, in contrast, dominant individuals can aggressively appropriate a resource, such joining behaviour becomes increasingly difficult to employ with decreasing social rank because adult individuals can only join others lower ranking than themselves. Our study supports theoretical predictions and highlights potentially important individual constraints on the ability of individuals of low social rank to use social information, driven by competition with dominant conspecifics over monopolisable resources. 
1 Switching spatial scale reveals dominance-dependent social

2

3

4

5

6

7

8

9

10

11

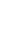

\section{foraging tactics in a wild primate}

(1)

7

8
${ }^{3}$ Centre of Excellence in Biological Interactions, Department of Biological and Environmental Sciences,
University of Jyväskylä, Jyväskylä, Finland

${ }^{1}$ Department of Zoology, University of Oxford, Oxford, UK

${ }^{2}$ The Institute of Zoology, Zoological Society of London, Regent's Park, London, UK

9

${ }^{*}$ Author for correspondence. Email address: allee@jyu.fi 


\section{Abstract}

13 When foraging in a social group, individuals are faced with the choice of sampling their

14 environment directly or exploiting the discoveries of others. The evolutionary dynamics of this

15 trade-off have been explored mathematically through the producer-scrounger game, which has

16 highlighted socially exploitative behaviours as a major potential cost of group living. However,

17 our understanding of the tight interplay that can exist between social dominance and

18 scrounging behaviour is limited. To date, only two theoretical studies have explored this

19 relationship systematically, demonstrating that because scrounging requires joining a

20 competitor at a resource, it should become exclusive to high-ranking individuals when

21 resources are monopolisable. In this study, we explore the predictions of this model through

22 observations of the natural social foraging behaviour of a wild population of chacma baboons

23 (Papio ursinus). We collected data through over 800 hours of focal follows of 101 adults and

24 juveniles across two troops over two 3-month periods. By recording over 7,900 social foraging

25 decisions at two spatial scales we show that, when resources are large and economically

26 indefensible, the joining behaviour required for scrounging can occur across all social ranks.

27 When, in contrast, dominant individuals can aggressively appropriate a resource, such joining

28 behaviour becomes increasingly difficult to employ with decreasing social rank because adult

29 individuals can only join others lower ranking than themselves. Our study supports theoretical

30 predictions and highlights potentially important individual constraints on the ability of

31 individuals of low social rank to use social information, driven by competition with dominant

32 conspecifics over monopolisable resources. 


\section{Introduction}

34 Socially exploitative behaviours occur when individuals make use of the resources of

35 competitors. A wide range of both theoretical and empirical studies over recent decades have

36 highlighted such behaviours as a major potential cost of group living (Giraldeau \& Dubois,

37 2008). Because resources such as food, mates, breeding territories, or safety from predation

38 generally show variation in their distribution through space or time, individuals should benefit

39 from gathering information about their local environment to improve decision-making (Valone,

40 1989, 2006; McNamara, Green \& Olsson, 2006). However, when the personal collection of

41 information requires search effort or risk taking, selection should favour the avoidance of these

42 costs through the collection and use of social information, where individuals attend to the

43 behaviours of others in a social group to exploit their efforts and knowledge (for review see

44 Valone \& Templeton, 2002; Danchin et al., 2004; Rieucau \& Giraldeau, 2011).

45

46 The dynamics of these interactions have been formalised as the producer-scrounger game

47 (Barnard \& Sibly, 1981; Barnard, 1984; Vickery et al., 1991). Supported by a wide range of

48 empirical studies (e.g., Koops \& Giraldeau, 1996; Mottley \& Giraldeau, 2000; Morand-Ferron,

49 Giraldeau \& Lefebvre, 2007), the producer-scrounger game has emerged as the prevailing

50 theoretical framework in which to study social foraging decisions (Vickery et al., 1991;

51 Giraldeau \& Caraco, 2000). In this game, producers actively search for resources, while

52 scroungers instead rely on social information to exploit the discoveries of producers. The two

53 tactics are considered mutually exclusive. Scrounging is thus under negative frequency-

54 dependent selection since its success, being dependent on the efforts of producers, is 
55 determined by the relative frequencies of the two tactics within a group. This dynamic is

56 expected to lead populations to an evolutionarily or behaviourally stable mix of producing and

57 scrounging (Giraldeau \& Dubois, 2008; Fawcett, Hamblin \& Giraldeau, 2013). As such,

58 scrounging behaviour has the potential to reduce the per capita rate of discovery of new

59 resources (Vickery et al., 1991), which may act to reduce average individual fitness in a

60 population (Coolen, Giraldeau \& Vickery, 2007).

61

62 The basic producer-scrounger model assumes that an individual's phenotype has no influence

63 on its decision or ability to play either tactic. All individuals are essentially equivalent, and are

64 expected to receive equal payoffs. However, many empirical studies have shown that an

65 individual's tactic choice may be strongly influenced or constrained by its phenotype (e.g.,

66 Beauchamp, 2001; Stahl et al., 2001; di Bitetti \& Janson, 2001; Kurvers et al., 2010). This has

67 potentially important fitness implications, since theory predicts that phenotype-limited games

68 may not reach an evolutionarily stable mix of strategies, resulting in differential payoffs across

69 individuals (Parker, 1982).

70

71 Since scrounging behaviour represents the exploitation of another's resource, one might expect

72 it to be strongly influenced by social dominance. Specifically, the competitive advantage of

73 high-ranking individuals should allow them to scrounge from others more easily (Parker, 1974;

74 Maynard Smith \& Parker, 1976; Hammerstein, 1981). Despite this expectation, empirical

75 studies have not been unanimous. While some experiments have demonstrated a clear positive

76 relationship between social dominance and scrounging behaviour (Stahl et al., 2001; Liker \& 
77 Barta, 2002; Lendvai, Liker \& Barta, 2006; McCormack, Jablonski \& Brown, 2007), a number of

78 other studies have not (Bugnyar \& Kotrschal, 2002; Robinette Ha \& Ha, 2003; Beauchamp,

79 2006; Teichroeb, White \& Chapman, 2015). This conflict might be reconciled by considering

80 more systematically the spatiotemporal distribution of resources faced by different taxa in both

81 naturalistic and experimental settings. The competitive benefits of social dominance are

82 expected to be associated with priority of access to resources, manifest as contest competition

83 (Kaufmann, 1983; Łomnicki, 2009). Consistent with this, resource defence theory predicts that

84 individuals should be more aggressive when defending a resource in accordance with both its

85 value and how easily it can be defended (Grant, 1993; Grant \& Guha, 1993; Robb \& Grant,

86 1998). Empirical studies into dominance and resource defence have demonstrated higher

87 foraging success for socially dominant individuals only when presented with limited food

88 patches that are monopolisable (Theimer, 1987; Vahl et al., 2005).

90 Some researchers have suggested that the integration of producer-scrounger and resource

91 defence theory might elucidate an interesting relationship between socially exploitative

92 behaviour and dominance (Barta \& Giraldeau, 1998; Giraldeau \& Dubois, 2008). Specifically,

93 dominant individuals should benefit disproportionately if they can use their competitive

94 advantage to ensure that only they can use social information effectively. Two studies (Barta \&

95 Giraldeau, 1998; Lee et al., 2016) have explored this hypothesis by modelling the effects of

96 between-individual asymmetries in competitive ability on producer-scrounger dynamics in a

97 group. They found that when social rank conferred no competitive advantage to an individual -

98 that is, resources were not monopolisable - groups converged on basic producer-scrounger 
99 equilibria in which all individuals behave equivalently and receive equal payoffs. In contrast,

100 when individuals could use their social rank to gain a competitive advantage in monopolising a

101 resource, scrounging behaviour was strongly associated with dominance, and dominant

102 individuals achieved the highest payoffs (Barta \& Giraldeau, 1998; Lee et al., 2016). The driving

103 force behind this pattern was the fact that scrounging behaviour requires that a competitor is

104 joined at a resource in space and time, forging a causal link between the degree to which

105 contest competition acts and constraints on an individual's ability to use social information (Lee

106 et al., 2016). However, to date there has been no attempt to test these predictions empirically,

107 either in the laboratory or under naturalistic conditions.

108

109 In this study, we explore a key prediction generated by the unification of producer-scrounger

110 and resource defence theories, namely that there should be a strong link between social

111 dominance and the scrounger tactic only when resources are monopolisable. We did this by

112 studying the natural social foraging decisions made by wild chacma baboons (Papio ursinus)

113 across two spatial scales that are expected to differ in the degree to which dominant individuals

114 can monopolise food. At the first spatial scale - the 'patch' - resource clumps were too large to

115 be monopolised independently, while at the second - the 'sub-patch' - resource clumps were

116 smaller and monopolisation was possible (see Methods for further details on how these spatial

117 scales were defined). Because our focus was on naturalistic behaviour, we did not manipulate

118 the information available to individuals while foraging to manufacture a situation where joining

119 a competitor always represented the exclusive use of social information, which could accurately

120 be termed scrounging (Vickery et al., 1991). Rather, we consider the observable joining 
121 behaviours of individuals as they foraged, representing competitive interactions fundamental to

122 the predictions of producer-scrounger theory (Lee et al., 2016). In this way, we explore the

123 competitive constraints that social rank may impose on an individual's ability to use social

124 information through joining behaviour, and the relation of such constraints to resource

125 monopolisability in a naturalistic setting in which information use is expected to be important.

126 We make the following three predictions: 1) joining behaviour should show a strong positive

127 association with dominance rank at the smaller, sub-patch level but not at the larger, patch

128 level; 2) individuals should join those to whom they are dominant at the sub-patch level, but

129 there should be no systematic asymmetry in dominance when joining occurs at the patch level;

130 and 3) joining should be associated with competitive exclusion (i.e., resource monopolisation)

131 at the sub-patch level, but not at the patch level.

132

133 Materials and Methods

134 Study Site and Species

135 Fieldwork was conducted at Tsaobis Nature Park, Namibia $\left(22^{\circ} 23^{\prime} \mathrm{S}, 15^{\circ} 45^{\prime} \mathrm{E}\right)$, during two three-

136 month periods between August and October in 2012 and 2013. Two groups of chacma

137 baboons, hereafter referred to as troop 'J' (group size and compositions: $N_{J, 2012}=54$, adult

138 females $=16$, adult males $=15$, juveniles $=23 ; N_{J, 2013}=58$, adult females $=18$, adult males $=9$,

139 juveniles $=31)$ and troop ' $\mathrm{L}$ ' $\left(N_{L, 2012}=51\right.$, adult females $=18$, adult males $=6$, juveniles $=27$;

$140 N_{L, 2013}=62$, adult females $=19$, adult males $=11$, juveniles $=32$ ), were the focus of study. All

141 baboons were individually recognisable and habituated to the presence of observers at close

142 proximity. Each group was followed daily from dawn until dusk (see Huchard et al., 2009 for 
143 further information). For each year, data were collected for all individual baboons $>6$ months of

144 age (the age at which young baboons begin to forage independently of their mother) at the

145 start of the study period, resulting in a total sample of 101 individuals (2012: 54 adults, 43

146 juveniles; 2013: 50 adults, 41 juveniles). Differences in the sample of individuals across years

147 were due to death, emigration, or passing the minimum age threshold.

148

149 Chacma baboons are an ideal model system for our study, since they live in large, stable social 150 groups in which linear dominance hierarchies are clear (Altmann \& Altmann, 1973), individuals 151 generally feed at the same time (King \& Cowlishaw, 2009), the use of social information while

152 foraging has been demonstrated in field-based experiments (Carter, Torrents Ticó \& Cowlishaw, 153 2016), and socially exploitative foraging interactions are common (King, Isaac \& Cowlishaw, 154 2009; Marshall et al., 2012). Furthermore, our study troops spent approximately 80\% of their 155 foraging time during the study period in a riparian woodland environment, characterised by 156 large trees including Faidherbia albida, Salvadora persica, Acacia erioloba, Acacia tortilis, and 157 Prosopis glandulosa. Within this feeding environment, we defined two spatial scales between 158 which the ability of dominant individuals to monopolise food were predicted to differ: the 159 patch and the sub-patch.

160

161 The patch represents the scale traditionally used in foraging theory and ecology, and is defined

162 as a spatially discrete unit of a food resource (Wiens, 1976). Here, we refined this definition 163 such that the operational definition of a patch was a single tree or shrub, or a collection of 164 conspecific trees or shrubs growing together with a continuous canopy separated by no more 
165 than $1 \mathrm{~m}$ (median surface area $=156 \mathrm{~m}^{2}$, interquartile range $=28-237 \mathrm{~m}^{2} ; n=59$; see Marshall

166 et al., 2012 for further details). In contrast, the sub-patch was defined as the area in a patch

167 within which an individual could feed without travelling (i.e., within arm's reach of a stationary

168 baboon, approximately $2.25 \mathrm{~m}^{2}$ ). This is equivalent to the 'feeding station' scale that has

169 received some attention in the foraging literature (Kotliar \& Wiens, 1990; see Searle, Hobbs \&

170 Shipley, 2005 for a review). Given the large size of patches compared with sub-patches,

171 dominant individuals should be able to competitively exclude subordinate others more easily at

172 the latter scale.

173

174 Data Collection and Processing

175 Information regarding individual social foraging decisions and interactions at each spatial scale

176 was recorded through focal sampling (Altmann, 1974) on Motorola ES400 Personal Digital

177 Assistants and Google Nexus 4 Smartphones using a customised data capture application in the

178 database-driven software Cybertracker v.3.317 (http://cybertracker.org). Focal follows lasted

179 between 15 and 30 minutes, and the same individual was not studied more than once within a

180 6-hour period. Individuals were selected for focal observation using a pseudorandom sampling

181 process, which ensured even coverage across different times of day (based on four consecutive

182 3-hour time blocks from 06:00 to 18:00) and different months.

183

184 A patch entry event was recorded whenever the focal individual searched for or consumed food

185 in a new patch for 5 seconds or more. While in a patch, the focal individual could move

186 between sub-patches. A sub-patch entry was recorded when an individual relocated into a new 
187 area of a patch to forage, and either remained stationary for $\geq 5$ seconds while standing, or sat 188 for $\geq 1$ second, to forage in this location. In this way, foraging behaviour at each spatial scale 189 was studied at the level of investment, since entries need not have resulted in successful food 190 consumption (although in almost all cases did). At each spatial scale, a specific foraging decision 191 was assigned to every entry event. The decision was defined as 'produce' if the patch or sub192 patch being entered was unoccupied, and 'join' if occupied by a conspecific. Note that join 193 events at the sub-patch level need not have been preceded by a join event at the patch level, 194 because 1) a focal individual could enter an unoccupied patch and subsequently be joined by 195 others, providing opportunities for future join events at the sub-patch level; and 2) focal follows 196 could begin with the focal individual already occupying a patch.

197

198 For each join event, the number and identity of individuals occupying the resource was 199 recorded. In cases where visibility was poor, a minimum number of occupants was estimated 200 and, where known, their identity recorded. Since individuals being joined could either remain 201 in, or be supplanted from, their patch or sub-patch, we recorded whether or not a join event 202 was associated with competitive exclusion. We defined supplanting, representing competitive exclusion, as an approach-retreat interaction (Rowell, 1966; Silk et al., 2010) at a given patch or sub-patch that resulted in the entry and exit of the approaching and retreating individuals, respectively.

207 Since the size of a patch is variable, while sub-patch size is fixed, the relationship between them 208 is such that at the smallest, or 'critical', patch sizes they reach equivalence. With this in mind, 
209 social foraging decisions were included only where a sub-patch structure could be defined (i.e.,

210 where the occupied patch held more than one sub-patch), such that the sub-patch always

211 represented a smaller spatial scale nested within the patch. Study at the sub-patch scale thus

212 captured social foraging dynamics at a resolution higher than at the patch scale, allowing us to

213 avoid conflating processes working at the two different spatial scales. Specifically,

214 monopolisation of food at the patch scale always required the defence of an area at least (but

215 generally considerably more than) double that required at the sub-patch scale. The data were

216 then filtered further to exclude all ambiguous foraging decisions that could not clearly be

217 classified as either produce or join (<10\%). A total of 801 focal hours were carried out across

218 the two study periods on 101 individual baboons (mean \pm s.e. $=7.9 \pm 0.1$ hours per individual),

219 resulting in a dataset of 1861 patch entry and 5050 sub-patch entry decisions for analysis. All

220 observers completed a period of intensive training in the field to ensure high levels of accuracy

221 and consistency in recognising patch and sub-patch boundaries, entry events, foraging

222 decisions, and competitive exclusion. Observers were also naïve to the predictions of the study

223 relating to associations between joining behaviour and social dominance at the two spatial

224 scales.

225

226 A dominance hierarchy was generated for each troop-year combination using pairwise agonistic

227 interactions occurring within each study period. These interactions were collected both during

228 focal follows and through ad libitum sampling, and were used to make actor-receiver matrices

229 indicating the number of agonistic interactions occurring between each dyad in each direction.

230 No dominance interactions occurring during foraging decisions were included in the matrices. In 
231 addition, all interactions involving individuals not yet weaned from their mother were excluded,

232 because dominance asymmetry at this age is strongly influenced by the mother's presence and

233 behaviour (Cheney, 1977). Each actor-receiver matrix $\left(N_{2012, J}=1010 ; N_{2012, L}=1025 ; N_{2013, J}=\right.$

234 833; $N_{2013, L}=1073$ ) was reordered using Matman 1.1.4 (Noldus Information Technology 2003),

235 optimised by selecting the hierarchy with the lowest level of conflict (i.e., minimising the

236 number of interactions inconsistent with the predicted hierarchy) using a heuristic search

237 algorithm with ten thousand randomisations. Linearity was supported for all four hierarchies

238 (Landau's corrected linearity index: $h_{2012, J}^{\prime}=0.19 ; h_{2012, L}^{\prime}=0.32 ; h_{2013, J}^{\prime}=0.18 ; h_{2013, L}^{\prime}=0.15, p<$

2390.001 in all cases), highlighting the rarity of interactions inconsistent with the predicted

240 hierarchy $\left(n_{2012, J}=67(7 \%) ; n_{2012, L}=81(8 \%) ; n_{2013, J}=43(5 \%) ; n_{2013, L}=71(7 \%)\right)$. Individuals not

241 yet weaned were then re-entered into the appropriate dominance hierarchy based on their

242 maternal rank (i.e., one position below their mother, consistent with the well-documented

243 maternal reinforcement of offspring rank in chacma baboons; Cheney, 1977), producing

244 complete hierarchies that included all members of the group for each year. To control for

245 differences in the size of groups within and across years, all absolute ranks (ranging from 1 to $n$ )

246 were standardised to between 0 (lowest rank) and 1 (highest rank) following 1-((1-r)/(1-n)),

247 where $r$ is the absolute rank of an individual.

248

249 Our wholly observational research adhered to the Guidelines for the Use of Animals in

250 Behavioural Research and Teaching (Animal Behaviour 2012. 83:301-309), and our protocols

251 were assessed and approved by the Ethics Committee of the Zoological Society of London 
252 (BPE/0518). Our study was approved by the Ministry of Environment and Tourism in Namibia

253 (Research Permits 1696/2012 and 1786/2013).

254

255 Statistical analyses

256 Our analysis was divided into three sections consistent with the three main predictions outlined

257 above. First, we used generalised linear mixed-effects modelling (GLMM) to explore how the

258 relationship between social dominance and joining behaviour changed across spatial scales due

259 to differences in resource monopolisability. Our main prediction was that all individuals would

260 exhibit joining behaviour at the patch scale, but that there would be a strong positive

261 relationship between rank and joining at the sub-patch scale. However, since juvenile baboons

262 are often tolerated at feeding sites (e.g., Huchard et al., 2013), we predicted that this positive

263 relationship (and thus an interaction between spatial scale and dominance rank) would only

264 hold for adults. While differences between adults and juveniles were not the focus of this study,

265 it was important to include age class in our statistical models to fully understand any

266 relationships between resource monopolisability, contest competition, and joining behaviour.

267 We thus constructed our statistical model with a three-way interaction between spatial scale

268 ('patch' or 'sub-patch'), dominance rank, and age class ('juvenile' or 'adult'). The response

269 variable was given as a binary indicator of the decision at each entry to either 'produce' or

270 'join', scored as 0 or 1 , respectively. We fit a binomial error structure to the GLMMs. Model

271 selection was conducted by using a likelihood ratio test $(\alpha=0.05)$ to judge whether the model

272 with or without the three-way interaction term provided the better fit to the data, and if the

273 latter, whether those models with or without two-way interactions between spatial scale, 
274 dominance rank, and age class provided the better fit. Troop and year were included as control

275 fixed effects, and were thus retained in all models. Focal identity and focal follow number were 276 included as random intercepts in all models.

277

278 Second, we asked whether join 'events' were consistently associated with asymmetries in social

279 rank at each spatial scale. Our main prediction was that individuals would consistently join

280 others lower ranked than themselves at the sub-patch level, but would join others regardless of

281 rank differences at the patch level. Again, we predicted that the relationship at the sub-patch

282 level would not hold for juvenile individuals. To test this second set of predictions, we used a

283 randomisation method to compare the joining behaviour we observed to the patterns of joining

284 that would be expected if individuals joined others randomly with respect to rank difference.

285 We employed this method because any relationship between dominance rank and joining

286 frequency demonstrated in our first analysis would indicate that a crude comparison of the

287 rank difference between joining and joined individuals could lead us to erroneous conclusions.

288 For example, if high ranked individuals joined more frequently than low ranked individuals at a

289 given spatial scale, our data would suggest that individuals on average joined others lower

290 ranked than themselves at this spatial scale in the case that their actual joining behaviour was

291 random with respect to rank difference, simply because individuals with above average social

292 rank necessarily have more individuals subordinate to them than dominant to them. Our

293 observation variable for this analysis was a binary indicator of whether the joining individual

294 was dominant or subordinate to the joined individual. For those events where multiple

295 individuals were joined in a patch (36\%) or sub-patch (2\%), the direction of their average rank 
296 difference with the focal individual was used. We generated expectations of rank differences

297 under random joining behaviour with respect to rank difference by randomly resampling from

298 the appropriate troop only the identity of the joined individual for each observed join event,

299 and calculating the difference in rank between the actual joiner and this randomly sampled

300 individual. We repeated this process 10,000 times to generate a distribution of the expected

301 proportion of join events in which the joining individual would be subordinate to the joined

302 individual if individuals joined randomly with respect to rank difference. We defined that our

303 observed estimates for the proportion of events with a subordinate joiner deviated from

304 random expectations when they fell outside of the $95 \%$ tolerance intervals of the random

305 distribution. We built four sets of random distributions to which we could compare our

306 observational estimates: one for adult joiners and one for juvenile joiners at both the patch and

307 the sub-patch scale.

308

309 Third, we built a GLMM to establish whether join events at different spatial scales were

310 associated with differences in the competitive exclusion experienced by the joined individual.

311 Competitive exclusion was modelled as a binary response variable: individuals were either

312 supplanted from the resource or were not. Fixed effects were included as an interaction

313 between spatial scale and age class, and were assessed using likelihood ratio tests as described

314 above. Since these data were not available for patch level decisions in 2012, only decisions from

3152013 were used in this analysis. Troop was included as a control fixed effect, and so was

316 retained in all models, and focal identity and focal follow number were included as random

317 intercepts. We predicted that joining behaviour in adults would cause competitive exclusion of 
318 the joined individual at the sub-patch but not the patch scale, and that joining behaviour in

319 juveniles would result in lower levels of competitive exclusion at the sub-patch scale compared

320 with adults.

321

322 All analyses were conducted in R version 3.0.2. using the Ime4 package (Bates et al., 2013; R

323 Core Team, 2013).

324

325 Results

326

Dominance and social foraging decisions at different spatial scales

327 At the patch scale, joining behaviour was common regardless of social rank (Table 1; Fig. 1a),

consistent with our predictions. Although there was some increase in joining with social

329

dominance in adults, even the lowest ranked individuals entered occupied patches

330

approximately 55\% (95\% confidence intervals: $41 \%$ and $67 \%$ ) of the time. While joining was in

331

general much less common at the sub-patch scale, there was a strong positive relationship in

332

adults between dominance and joining behaviour (twice that at the patch scale) that was

333

consistent with our predictions (Table 1; Fig. 1b). The lowest ranked adults had around a 1\%

334

probability (95\% confidence intervals: $0 \%$ and $2 \%$ ) of joining when entering a new sub-patch,

335

while mid-ranked and top-ranked adults did so approximately $4 \%$ (95\% confidence intervals: $3 \%$

336

and $6 \%$ ) and $11 \%$ (95\% confidence intervals: $8 \%$ and $17 \%$ ) of the time, respectively.

337

338 As predicted, the effects of social rank on the probability of joining were weaker in juveniles,

339 and this held across both spatial scales such that there was in general no relationship between 
340 dominance and joining frequency in juveniles (Table 1; Fig.1). In addition, juveniles were on

341 average more likely than adults to join at the patch scale, but this primarily reflected low-

342 ranking juveniles joining much more frequently than similarly ranked adults when entering a

343 new patch. This effect was somewhat weakened at the sub-patch scale: low-ranked juveniles

344 joined more frequently when entering a new sub-patch than similarly ranked adults but the 345 pattern was reversed for high-ranked juveniles.

347 Combined, our results reflect support for the three possible two-way interactions between

348 spatial scale, dominance rank, and age class (Table 1). Our lack of support for a three-way

349 interaction between these effects (likelihood ratio test: $\chi^{2}=0.70, p=0.41$ ) reflects the fact that

350 there was some increase in joining with social rank in adults at both spatial scales. This meant

351 that a stronger effect of dominance in adults at the sub-patch versus patch level, combined

352 with no clear relationship between joining frequency and social rank in juveniles at either

353 spatial scale, was captured by a general (i.e., not age class-specific) increase in joining with

354 dominance at the sub-patch level and a general (i.e., not spatial scale-specific) decrease in the 355 effect of dominance on joining in juveniles.

357 Social constraints on joining behaviour at different spatial scales

358 At the patch scale, there was no evidence that adult individuals joined others systematically

359 higher or lower ranked than themselves compared with the rank asymmetries expected if 360 individuals joined others randomly with respect to their rank difference (Fig.2a, $N_{\text {patch,adult }}=$

361 313). Although adults were less likely to join individuals dominant to them at this spatial scale, 
362 this could be explained under joining behaviour that was random with respect to rank

363 difference by the finding in our first analysis that the frequency of joining behaviour increased

364 slightly with increasing rank (Fig.1a).

365

366 At the sub-patch scale, adult individuals joined individuals dominant to themselves in $9 \%$ of join

367 events, and so were less likely to do so than would have been expected if individuals joined

368 others randomly with respect to rank difference (Fig.2b; $N_{\text {sub-patch, } \text { adult }}=154$ ) and given the fact

369 that high ranked individuals are much more likely than low ranked individuals to join others at

370 this spatial scale (Fig.1b). We thus found support for our prediction that adult individuals would

371 consistently join others lower ranked than themselves at the sub-patch level.

372

373 Comparisons between observed and random joining behaviour for juveniles were broadly

374 similar to those for adults. At the patch scale, the rank asymmetries at observed join events did

375 not deviate from expectations under joining behaviour random with respect to rank difference

376 (Fig. 2c; $N_{\text {patch,juvenile }}=349$ ). At the sub-patch scale, we observed juveniles joining others to

377 whom they were subordinate in $49 \%$ of cases. Although this shows that juveniles were

378 frequently able to join others dominant to themselves, they nonetheless did so less often than

379 expected under random joining (Fig. 2d; $N_{\text {sub-patch,juvenile }}=147$ ). Despite the fact that our first

380 analysis found no relationship between dominance and joining frequency for juveniles at the

381 sub-patch level (Fig.1b), the random distribution at this spatial scale reflects the fact that

382 juveniles are usually below average in rank. 
384 Competitive exclusion at different spatial scales

385 Joining caused competitive exclusion at the sub-patch scale much more than it did at the patch

386 scale (Table 1). In adults, joining at the patch scale was associated with competitive exclusion in

$3879 \%$ of cases. This figure increased to $79 \%$ at the sub-patch scale. Joining by juveniles was less

388 likely to result in competitive exclusion at both spatial scales (patch: 3\%; sub-patch: 51\%).

389

390 Discussion

391 We provide empirical evidence that joining behaviour should be more strongly related to social

392 rank when the competitive asymmetries associated with dominance are stronger, in support of

393 previous theoretical predictions (Barta \& Giraldeau, 1998; Lee et al., 2016). We show that

394 changes in resource monopolisability can mediate this shift in competitive asymmetry through

395 changes in competitive exclusion at different spatial scales. At the larger patch scale, adults

396 could join others regardless of any differences in their social ranks, and did so frequently. At the

397 smaller sub-patch scale, joining was a rarer event - likely reflecting the higher finder's share at

398 this spatial scale (Vickery et al., 1991) - but also represented a more exclusive tactic, almost

399 non-existent in the lowest-ranking individuals but increasing in probability with social

400 dominance. When resources are monopolisable, socially subordinate individuals may thus be

401 constrained in their ability to exploit available social information when its use requires joining

402 behaviour, as is assumed by producer-scrounger theory. Since the size of a resource is expected

403 to influence its economic defensibility (Grant, 1993), our study supports calls to unify producer-

404 scrounger and resource defence theory in order to better understand the relationship between

405 dominance and socially exploitative behaviours (Barta \& Giraldeau, 1998; Dubois, Giraldeau \& 
406 Grant, 2003; Dubois \& Giraldeau, 2005; Giraldeau \& Dubois, 2008). However, our study also

407 highlights two areas in which our understanding of the evolutionary ecology of animal

408 information use is still lacking, requiring further theoretical and empirical developments.

409

410 First, we showed a strong increase in the frequency of joining at higher ranks for adults at the

411 sub-patch scale, where single individuals could use their dominance to exclude competitors.

412 However, the pattern we observed was weaker than that predicted by Barta and Giraldeau

413 (1998), who suggested a complete absence of scrounging in all but the most dominant

414 individuals when resources could be effectively monopolised. We found that even middle- and

415 low-ranking adults can scrounge from others, provided that the others they join are even lower

416 ranked than themselves. The failure of the producer-scrounger model to predict this pattern

417 likely reflects two of its assumptions. Specifically, the model is built such that any individual

418 playing scrounger can access all the discoveries of others, since (1) resources are assumed to be

419 so rare that they are discovered one-at-a-time and (2) scroungers can access perfect social

420 information and so detect each discovery (Vickery et al. 1991b; Giraldeau \& Caraco 2000; cf.

421 Ohtsuka \& Toquenaga 2009). When resources can be monopolised, these conditions mean that

422 only the highest-ranked individuals will benefit from scrounging behaviour. However, in many

423 groups of social foragers multiple patches can be discovered at the same time (and

424 simultaneous discoveries or options will likely be the norm for many other types of resource

425 too). As such, scrounging individuals will be unable to access all resource discoveries even if

426 they have perfect social information (Ohtsuka \& Toquenaga, 2009; Afshar \& Giraldeau, 2014).

427 Furthermore, since individuals within social groups are unlikely to be in close proximity at all 
428 times (Krause \& Ruxton, 2002; for this study population, see Cowlishaw, 1999; Castles et al.,

429 2014), scrounging individuals are unlikely to possess perfect social information regarding all

430 discoveries occurring at the group level (Barta, Flynn \& Giraldeau, 1997; Hirsch, 2007), and any

431 temporal and energetic costs associated with scrounging may vary both within and between

432 individuals.

433

434 Under the conditions of simultaneous discoveries and imperfect access to social information,

435 the most dominant individuals will be unable to monopolise all resource discoveries, regardless

436 of the economic defensibility of single resource patches. Instead, as shown here, the difference

437 in rank between individuals across a hierarchy should play an important role in mediating

438 scrounging behaviour. This finding is consistent with the predictions of a recent game theoretic

439 model (Lee et al., 2016), which proposes that if the highest ranking animals in a group do not

440 detect a particular patch discovery, and/or are occupied at another discovery, then middle and

441 lower ranking animals can benefit from scrounging, provided that the producers from whom

442 they are scrounging are lower ranking than themselves. Our result that joining at the sub-patch

443 level still increases with social rank likely reflects the fact that higher ranked individuals have

444 more competitors who are subordinate to them. The most dominant individual should thus be

445 unconstrained in its ability to act upon opportunities to scrounge, and constraints should

446 increase down the dominance hierarchy. Furthermore, theory predicts that if more dominant

447 individuals are more effective at monopolising other's resource discoveries, then they should

448 continue to scrounge even under conditions that drive lower-ranked individuals to switch to the

449 producer tactic (e.g., when the finder's share is large; Lee et al., 2016). Individuals may also 
450 benefit from positioning themselves so as to maximise scrounging opportunities (Barta, Flynn \&

451 Giraldeau, 1997; di Bitetti \& Janson, 2001; Hirsch, 2007). Since dominant individuals may be

452 better able to secure more central positions in the group, this may improve their ability to

453 detect, and increase their proximity to, the discoveries of others (Barta, Flynn \& Giraldeau,

454 1997; King, Isaac \& Cowlishaw, 2009), and further reinforce the effects of dominance on

455 scrounging behaviour.

456

457 Second, we showed that the relationship between social dominance and joining is strongly

458 influenced by age class. In stark contrast to adults, juvenile baboons showed no general

459 relationship between social dominance and joining, particularly at the sub-patch level. While an

460 adult baboon's ability to join competitors at this spatial scale depended strongly on their

461 relative dominance, juveniles were less constrained by their social status. Indeed, juvenile

462 behaviour accounted for almost all instances where a subordinate joined a higher-ranking

463 individual at the sub-patch level. There are two likely explanations for this pattern. Firstly, rank

464 acquisition in chacma baboons is mediated primarily through maternal reinforcement.

465 Specifically, a mother will use aggressive behaviours to establish the dominance of her

466 developing offspring over others subordinate to her (Cheney, 1977; Holekamp \& Smale, 1991;

467 Lea et al., 2014). Consequently, social rank during early life might be particularly sensitive to

468 context, such that social interactions between juveniles may only reflect rank differences

469 between their mothers in the presence of the dominant mother. In the absence of the

470 dominant mother, older but 'subordinate' juveniles might use their larger size to join younger,

471 smaller, but more 'dominant' competitors. Secondly, there is evidence that juvenile baboons, 
472 like the juveniles of several other primate species (Janson, 1985), are more frequently tolerated

473 at feeding sites than adults. The presence of co-foraging juveniles may impose only a minimal

474 direct cost to adults, but permitting close kin access to resources may provide inclusive fitness

475 benefits. In particular, father-offspring relationships in chacma baboons afford juveniles access

476 to high-quality feeding sites (Huchard et al., 2013). Such toleration may mean that, in addition

477 to better access to monopolisable resources, low-ranked juveniles may not be constrained in

478 their ability to use social information in the same way that similarly ranked adults will be. Our

479 findings do not provide strong evidence in support of either tolerance or juvenile rank

480 instability in disrupting the expected positive relationship between social rank and joining

481 behaviour when resources are monopolisable. However, we might expect juvenile rank stability

482 to disrupt rank asymmetries but still to involve competitive exclusion when resources are

483 monopolisable, while toleration of juveniles should result in reduced levels of competitive

484 exclusion. The fact that the probability of competitive exclusion was reduced for juveniles

485 compared with adults at the sub-patch scale might suggest that toleration is playing a bigger

486 role than juvenile rank instability in our observations.

487

488 Our study demonstrates the way in which the monopolisability of resources may drive social 489 constraints on a subordinate individual's ability to use joining behaviour to access them. We 490 also show that such constraints may be relaxed in juveniles. Since resources generally show 491 some uncertainty in their distribution through space and time, information use is likely to play a 492 key role in resource acquisition. Our study illustrates how competitive processes associated 493 with dominance might facilitate or constrain an individual's ability to benefit from collecting 
494 social information when its use requires joining behaviour, as in the producer-scrounger

495 framework (Barta \& Giraldeau, 1998; Lee et al., 2016). An important step in future research will

496 be to develop frameworks that simultaneously consider how resource distributions underpin 1)

497 the strength and type of competition between individuals, 2) the benefits of collecting

498 information socially versus personally, and 3) the rate at which such information becomes out-

499 dated. This approach will elucidate the environmental conditions that should generate

500 interdependencies between contest competition and social information use by highlighting

501 when social information use should be dependent on joining behaviour at a resource. These

502 insights will allow better characterisation of the ways in which competition can modulate

503 relationships between an individual's ability to use information and its access to resources, and

504 the implications of such modulations for the dynamics of natural populations. 


\section{Acknowledgements}

506 We would like to say a big thank you to James Ounsley, Cassandra Raby, Rebecca Boulton, 507 Matthis Petit, Eveline Rijksen, Miles Keighley, Maddie Castles, Stef Oberprieler, Alice Baniel, 508 Stella Diamant, Katie Hatton, Julien Collet, Chris Smith, Boris Granovskiy, Caitlin Miller, Alecia

509 Carter, Elise Huchard, Hannah Wilmot, and Willem Odendaal for their work and support in the 510 field. Thanks also to Tim Coulson, Marcus Rowcliffe, Ben Sheldon, David Macdonald, Daniel van

511 der Post, Andrés López-Sepulcre, Alexander Weiss, and three anonymous reviewers for

512 constructive comments and discussions. Permission to work at the field site was kindly granted

513 by Tsaobis Nature Park, the Wittreich and Snyman families, and the Ministry of Lands and

514 Resettlement. We also thank the Gobabeb Training and Research Centre for affiliation in

515 Namibia. This study is a publication of the ZSL Institute of Zoology's Tsaobis Baboon Project. 


\section{References}

517 Afshar M., Giraldeau L-A. 2014. A unified modelling approach for producer-scrounger games in 518 complex ecological conditions. Animal Behaviour 96:167-176. DOI:

519 10.1016/j.anbehav.2014.07.022.

520

Altmann J. 1974. Observational Study of Behavior: Sampling Methods. Behaviour 49:227-265.

521 DOI: $10.1163 / 156853974 \times 00534$.

\section{2}

Altmann SA., Altmann J. 1973. Baboon ecology. Chicago, IL: University of Chicago Press.

Barnard CJ. 1984. Producers and scroungers: strategies of exploitation and parasitism. London, $524 \quad$ UK: Chapman \& Hall.

525 Barnard CJ., Sibly RM. 1981. Producers and scroungers: a general model and its application to 526 captive flocks of house sparrows. Animal Behaviour 29:543-550. DOI: 10.1016/S0003$527 \quad 3472(81) 80117-0$.

528 Barta Z., Flynn R., Giraldeau L-A. 1997. Geometry for a selfish foraging group: a genetic 529 algorithm approach. Proceedings of the Royal Society B: Biological Sciences 264:1233530 1238. DOI: 10.1098/rspb.1997.0170.

531 Barta Z., Giraldeau L-A. 1998. The effect of dominance hierarchy on the use of alternative 532 foraging tactics: a phenotype-limited producing-scrounging game. Behavioral Ecology and $533 \quad$ Sociobiology 42:217-223. DOI: 10.1007/s002650050433.

534 Bates D., Maechler M., Bolker B., Walker S. 2013. Ime4: Linear mixed-effects models using Eigen 535 and S4. R package version 1.0-5. 
536 Beauchamp G. 2001. Consistency and flexibility in the scrounging behaviour of zebra finches. 537 Canadian Journal of Zoology 79:540-544. DOI: 10.1139/cjz-79-3-540.

538 Beauchamp G. 2006. Phenotypic Correlates of Scrounging Behavior in Zebra Finches: Role of 539 Foraging Efficiency and Dominance. Ethology 112:873-878. DOI: 10.1111/j.1439$540 \quad$ 0310.2006.01241.x.

541 di Bitetti MS., Janson CH. 2001. Social foraging and the finder's share in capuchin monkeys, 542 Cebus apella. Animal Behaviour 62:47-56. DOI: 10.1006/anbe.2000.1730.

543 Bugnyar T., Kotrschal K. 2002. Scrounging tactics in free-ranging ravens, Corvus corax. Ethology 544 108:993-1009. DOI: 10.1046/j.1439-0310.2002.00832.x.

545 Carter A., Torrents Ticó M., Cowlishaw G. 2016. Sequential phenotypic constraints on social 546 information use in wild baboons. eLife:1-21. DOI: 10.7554/eLife.13125.

547 Castles M., Heinsohn R., Marshall HH., Lee AEG., Cowlishaw G., Carter AJ. 2014. Social networks 548 created with different techniques are not comparable. Animal Behaviour 96:59-67. DOI:

$549 \quad$ 10.1016/j.anbehav.2014.07.023.

550 Cheney DL. 1977. The acquisition of rank and the development of reciprocal alliances among

551 free-ranging immature baboons. Behavioral Ecology and Sociobiology 2:303-318. DOI:

$552 \quad$ 10.1007/BF00299742.

553 Coolen I., Giraldeau L-A., Vickery W. 2007. Scrounging behavior regulates population dynamics.

554 Oikos 116:533-539. DOI: 10.1111/j.2006.0030-1299.15213.x.

555 Cowlishaw G. 1999. Ecological and social determinants of spacing behaviour in desert baboon 

groups. Behavioral Ecology and Sociobiology 45:67-77. DOI: 10.1007/s002650050540.

557 Danchin E., Giraldeau L-A., Valone TJ., Wagner RH. 2004. Public information: from nosy 558 neighbors to cultural evolution. Science 305:487-491. DOI: 10.1126/science.1098254.

559 Dubois F., Giraldeau L-A. 2005. Fighting for resources: the economics of defense and 560 appropriation. Ecology 86:3-11. DOI: 10.1890/04-0566.

561 Dubois F., Giraldeau L-A., Grant JWA. 2003. Resource defense in a group-foraging context. 562 Behavioral Ecology 14:2-9. DOI: 10.1093/beheco/14.1.2.

563 Fawcett TW., Hamblin S., Giraldeau L-A. 2013. Exposing the behavioral gambit: The evolution of 564 learning and decision rules. Behavioral Ecology 24:2-11. DOI: 10.1093/beheco/ars085.

565 Giraldeau L-A., Caraco T. 2000. Social foraging theory. Princeton, NJ: Princeton University Press.

566 Giraldeau L-A., Dubois F. 2008. Social Foraging and the Study of Exploitative Behavior. Advances 567 in the Study of Behavior 38:59-104. DOI: 10.1016/S0065-3454(08)00002-8.

568 Grant JWA. 1993. Whether or not to defend? The influence of resource distribution. Marine 569 and Freshwater Behaviour and Physiology 23:137-153. DOI:

$570 \quad 10.1080 / 10236249309378862$.

571 Grant JWA., Guha RT. 1993. Spatial clumping of food increases its monopolization and defense 572 by convict cichlids, Cichlasoma nigrofasciatum. Behavioral Ecology 4:293-296. DOI:

$573 \quad 10.1093 /$ beheco/4.4.293.

574 Hammerstein P. 1981. The role of asymmetries in animal contests. Animal Behaviour 29:193575 205. DOI: 10.1016/S0003-3472(81)80166-2. 
576 Hirsch BT. 2007. Costs and benefits of within-group spatial position: a feeding competition 577 model. The Quarterly review of biology 82:9-27. DOI: 10.1086/511657.

578 Holekamp KE., Smale L. 1991. Dominance Acquisition During Mammalian Social Development:

579 The "Inheritance" of Maternal Rank. American Zoologist 317:306-317. DOI:

$580 \quad 10.1093 / \mathrm{icb} / 31.2 .306$

581 Huchard E., Alvergne A., Féjan D., Knapp LA., Cowlishaw G., Raymond M. 2009. More than 582 friends? Behavioural and genetic aspects of heterosexual associations in wild chacma 583 baboons. Behavioral Ecology and Sociobiology 64:769-781. DOI: 10.1007/s00265-009$584 \quad$ 0894-3.

585 Huchard E., Charpentier MJ., Marshall HH., King AJ., Knapp LA., Cowlishaw G. 2013. Paternal 586 effects on access to resources in a promiscuous primate society. Behavioral Ecology $587 \quad 24: 229-236$. DOI: 10.1093/beheco/ars158.

588 Janson $\mathrm{CH}$. 1985. Aggressive competition and individual food consumption in wild brown 589 capuchin monkeys (Cebus apella). Behavioral Ecology and Sociobiology 18:125-138. DOI: $590 \quad 10.1007 / B F 00299041$.

591 Kaufmann J. 1983. On The Defnintions and Functions of Dominance and Territoriality. Biology 592 Review 58:1-20. DOI: 10.1111/j.1469-185X.1983.tb00379.x.

593 King AJ., Cowlishaw G. 2009. All together now: behavioural synchrony in baboons. Animal 594 Behaviour 78:1381-1387. DOI: 10.1016/j.anbehav.2009.09.009.

595 King AJ., Isaac NJB., Cowlishaw G. 2009. Ecological, social, and reproductive factors shape 
596

597

598

599

600

601

602

603

604

605

606

607

608

609

610

611

612

613

614

615

producer-scrounger dynamics in baboons. Behavioral Ecology 20:1039-1049. DOI: 10.1093/beheco/arp095.

Koops MA., Giraldeau L-A. 1996. Producer-scrounger foraging games in starlings: a test of ratemaximizing and risk-sensitive models. Animal Behaviour 51:773-783. DOI: 10.1006/anbe.1996.0082.

Kotliar NB., Wiens JA. 1990. Multiple scales of patchiness and patch structure: a hierarchical framework for the study of heterogeneity. Oikos 59:253-260.

Krause J., Ruxton GD. 2002. Living in groups. Oxford, UK: Oxford University Press.

Kurvers RHJM., van Oers K., Nolet BA., Jonker RM., van Wieren SE., Prins HHT., Ydenberg RC. 2010. Personality predicts the use of social information. Ecology letters 13:829-37. DOI: 10.1111/j.1461-0248.2010.01473.x.

Lea AJ., Learn NH., Theus MJ., Altmann J., Alberts SC. 2014. Complex sources of variance in female dominance rank in a nepotistic society. Animal Behaviour 94:87-99. DOI: 10.1016/j.anbehav.2014.05.019.

Lee AEG., Ounsley JP., Coulson T., Rowcliffe JM., Cowlishaw G. 2016. Information use and resource competition: an integrative framework. Proceedings of the Royal Society B: Biological Sciences:20152550. DOI: http://dx.doi.org/10.1098/rspb.2015.2550.

Lendvai ÁZ., Liker A., Barta Z. 2006. The effects of energy reserves and dominance on the use of social-foraging strategies in the house sparrow. Animal Behaviour 72:747-752. DOI: 10.1016/j.anbehav.2005.10.032. 
616 Liker A., Barta Z. 2002. The effects of dominance on social foraging tactic use in house sparrows.

617 Behaviour 139:1061-1076. DOI: 10.1163/15685390260337903.

618 Łomnicki A. 2009. Scramble and contest competition, unequal resource allocation, and resource

619 monopolization as determinants of population dynamics. Evolutionary Ecology Research

$620 \quad 11: 371-380$.

621 Marshall HH., Carter AJ., Coulson T., Rowcliffe JM., Cowlishaw G. 2012. Exploring foraging

622 decisions in a social primate using discrete-choice models. The American Naturalist

623 180:481-95. DOI: 10.1086/667587.

624 Maynard Smith J., Parker GA. 1976. The logic of asymmetric contests. Animal Behaviour

625 24:159-175. DOI: 10.1016/S0003-3472(76)80110-8.

626 McCormack JE., Jablonski PG., Brown JL. 2007. Producer-scrounger roles and joining based on

627 dominance in a free-living group of Mexican jays (Aphelocoma ultramarina). Behaviour

628 144:967-982. DOI: 10.1163/156853907781492717.

629 McNamara JM., Green RF., Olsson O. 2006. Bayes' theorem and its applications in animal

630 behaviour. Oikos 112:243-251. DOI: 10.1111/j.0030-1299.2006.14228.x.

631 Morand-Ferron J., Giraldeau L-A., Lefebvre L. 2007. Wild Carib grackles play a producer

632 scrounger game. Behavioral Ecology 18:916-921. DOI: 10.1093/beheco/arm058.

633 Mottley K., Giraldeau L-A. 2000. Experimental evidence that group foragers can converge on

634 predicted producer-scrounger equilibria. Animal Behaviour 60:341-350. DOI:

$635 \quad$ 10.1006/anbe.2000.1474. 
636 Ohtsuka Y., Toquenaga Y. 2009. The patch distributed producer-scrounger game. Journal of 637 Theoretical Biology 260:261-266. DOI: 10.1016/j.jtbi.2009.06.002.

638 Parker GA. 1974. Assessment strategy and the evolution of fighting behaviour. Journal of 639 Theoretical Biology 47:223-243. DOI: 10.1016/0022-5193(74)90111-8.

640 Parker GA. 1982. Phenotype-limited evolutionarily stable strategies. In: King's College 641 Sociobiology Group ed. Current Problems in Sociobiology. Cambridge, UK: Cambridge $642 \quad$ University Press, 173-201.

643 R Core Team. 2013. R: A language and environment for statistical computing. Vienna, Austria: R $644 \quad$ Foundation for Statistical Computing.

645 Rieucau G., Giraldeau L-A. 2011. Exploring the costs and benefits of social information use: an 646 appraisal of current experimental evidence. Philosophical Transactions of the Royal Society 647 B: Biological Sciences 366:949-957. DOI: 10.1098/rstb.2010.0325.

648 Robb SE., Grant JWA. 1998. Interactions between the spatial and temporal clumping of food 649 affect the intensity of aggression in Japanese medaka. Animal Behaviour 56:29-34. DOI: $650 \quad$ 10.1006/anbe.1998.0735.

651 Robinette Ha R., Ha JC. 2003. Effects of ecology and prey characteristics on the use of 652 alternative social foraging tactics in crows, Corvus caurinus. Animal Behaviour 66:309-316. 653 DOI: 10.1006/anbe.2003.2182.

654 Rowell TE. 1966. Hierarchy in the organization of a captive baboon group. Animal Behaviour 655 14:430-443. DOI: 10.1016/S0003-3472(66)80042-8. 
656 Searle KR., Hobbs NT., Shipley LA. 2005. Should I stay or should I go ? Patch departure decisions 657 by herbivores at multiple scales. Oikos 111:417-424. DOI: 10.1111/j.0030-

658 1299.2005.13918.x.

659

660

661

662

663

664 665 666 667 668 669 670 671 672 673 674 675

Silk JB., Beehner JC., Bergman TJ., Crockford C., Engh AL., Moscovice LR., Wittig RM., Seyfarth RM., Cheney DL. 2010. Strong and consistent social bonds enhance the longevity of female baboons. Current biology : CB 20:1359-61. DOI: 10.1016/j.cub.2010.05.067.

Stahl J., Tolsma PH., Loonen MJJE., Drent RH. 2001. Subordinates explore but dominants profit: resource competition in high Arctic barnacle goose flocks. Animal behaviour 61:257-264. DOI: $10.1006 /$ anbe.2000.1564.

Teichroeb JA., White MMJ., Chapman CA. 2015. Vervet (Chlorocebus pygerythrus) Intragroup Spatial Positioning: Dominants Trade-Off Predation Risk for Increased Food Acquisition. International Journal of Primatology 36:154-176. DOI: 10.1007/s10764-015-9818-4.

Theimer TC. 1987. The effect of seed dispersion on the foraging success of dominant and subordinate dark-eyed juncos, Junco hyemalis. Animal Behaviour 35:1883-1890. DOI: 10.1016/S0003-3472(87)80081-7.

Vahl WK., Lok T., van der Meer J., Piersma T., Weissing FJ. 2005. Spatial clumping of food and social dominance affect interference competition among ruddy turnstones. Behavioral Ecology 16:834-844. DOI: 10.1093/beheco/ari067.

Valone TJ. 1989. Group foraging, public information, and patch estimation. Oikos 56:357-363. DOI: $10.2307 / 3565621$. 
676 Valone TJ. 2006. Are animals capable of Bayesian updating? An empirical review. Oikos

677 112:252-259. DOI: 10.1111/j.0030-1299.2006.13465.x.

678 Valone TJ., Templeton JJ. 2002. Public information for the assessment of quality: a widespread 679 social phenomenon. Philosophical Transactions of the Royal Society B: Biological Sciences $680 \quad 357: 1549-1557$. DOI: 10.1098/rstb.2002.1064.

681 Vickery WL., Giraldeau L-A., Templeton JJ., Kramer DL., Chapman CA. 1991. Producers, 682 scroungers, and group foraging. The American Naturalist 137:847-863. DOI:

$683 \quad$ 10.1006/anbe.1996.0014.

684 Wiens JA. 1976. Population Responses to Patchy Environments. Annual Review of Ecology and 685 Systematics 7:81-120. DOI: 10.1146/annurev.es.07.110176.000501.

686 


\section{Table $\mathbf{1}$ (on next page)}

Factors predicting the probability of joining behaviour and competitive exclusion associated with joining. 


\begin{tabular}{|c|c|c|c|c|c|c|}
\hline Response & $N$ & Fixed Effect & $\beta$ & s.e. & $\chi^{2}$ & $p$ \\
\hline Probability of & 6911 & Intercept & -0.05 & 0.26 & & \\
\hline \multirow[t]{9}{*}{ joining } & & Spatial scale (Sub-patch) & -4.51 & 0.28 & & \\
\hline & & Rank & 1.10 & 0.38 & & \\
\hline & & Age class (Juvenile) & 1.57 & 0.30 & & \\
\hline & & Troop (L) & 0.20 & 0.15 & & \\
\hline & & Year (2013) & 0.27 & 0.12 & & \\
\hline & & Spatial scale (Sub-patch) * Rank & 1.18 & 0.37 & 10.30 & 0.001 \\
\hline & & Rank * Age class (Juvenile) & -1.68 & 0.54 & 9.03 & 0.003 \\
\hline & & Spatial scale (Sub-patch) * Age & -0.68 & 0.21 & 10.40 & 0.001 \\
\hline & & class (Juvenile) & & & & \\
\hline Probability of & 385 & Intercept & -2.44 & 0.47 & & \\
\hline competitive & & Spatial scale (Sub-patch) & 4.55 & 0.65 & 164.27 & $<0.001$ \\
\hline \multirow[t]{2}{*}{ exclusion } & & Age class (Juvenile) & -1.61 & 0.47 & 14.06 & $<0.001$ \\
\hline & & Troop & -0.89 & 0.44 & & \\
\hline
\end{tabular}

Model reference categories: Spatial scale (Patch), Age class (Adult), Troop (J), Year (2012). 


\section{Figure 1 (on next page)}

Predicted relationship between the probability of joining behaviour and dominance rank at the (a) patch and (b) sub-patch level.

For both panels, solid and dashed lines represent the predicted values for adults and juveniles, respectively. Shaded green (adults) and purple (juveniles) regions are bounded by upper and lower $95 \%$ confidence intervals. Note the difference in scale of the two y-axes, reflecting the much lower levels of joining across all individuals at the sub-patch compared with patch scale. 


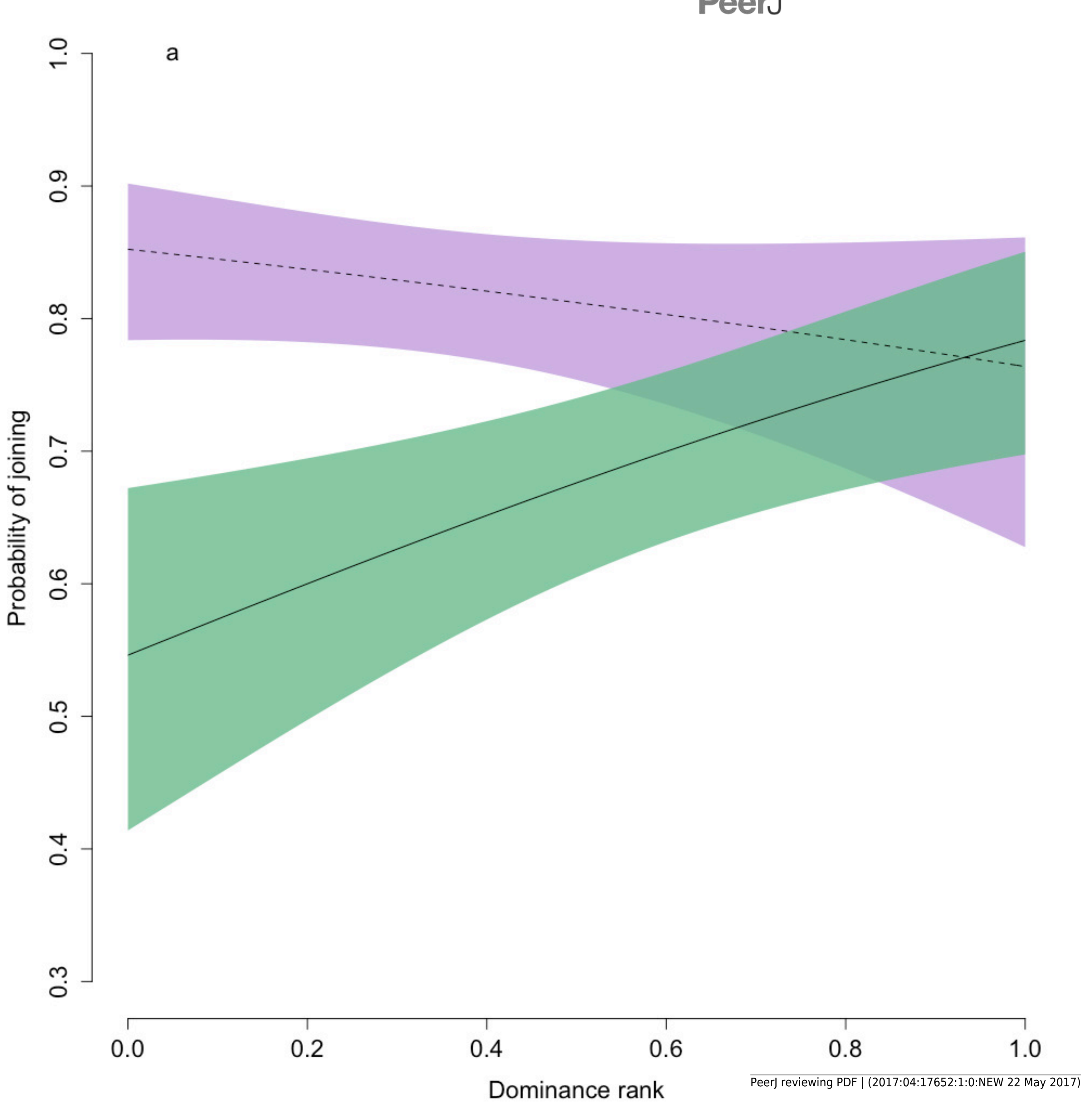

Manuscript to be reviewed

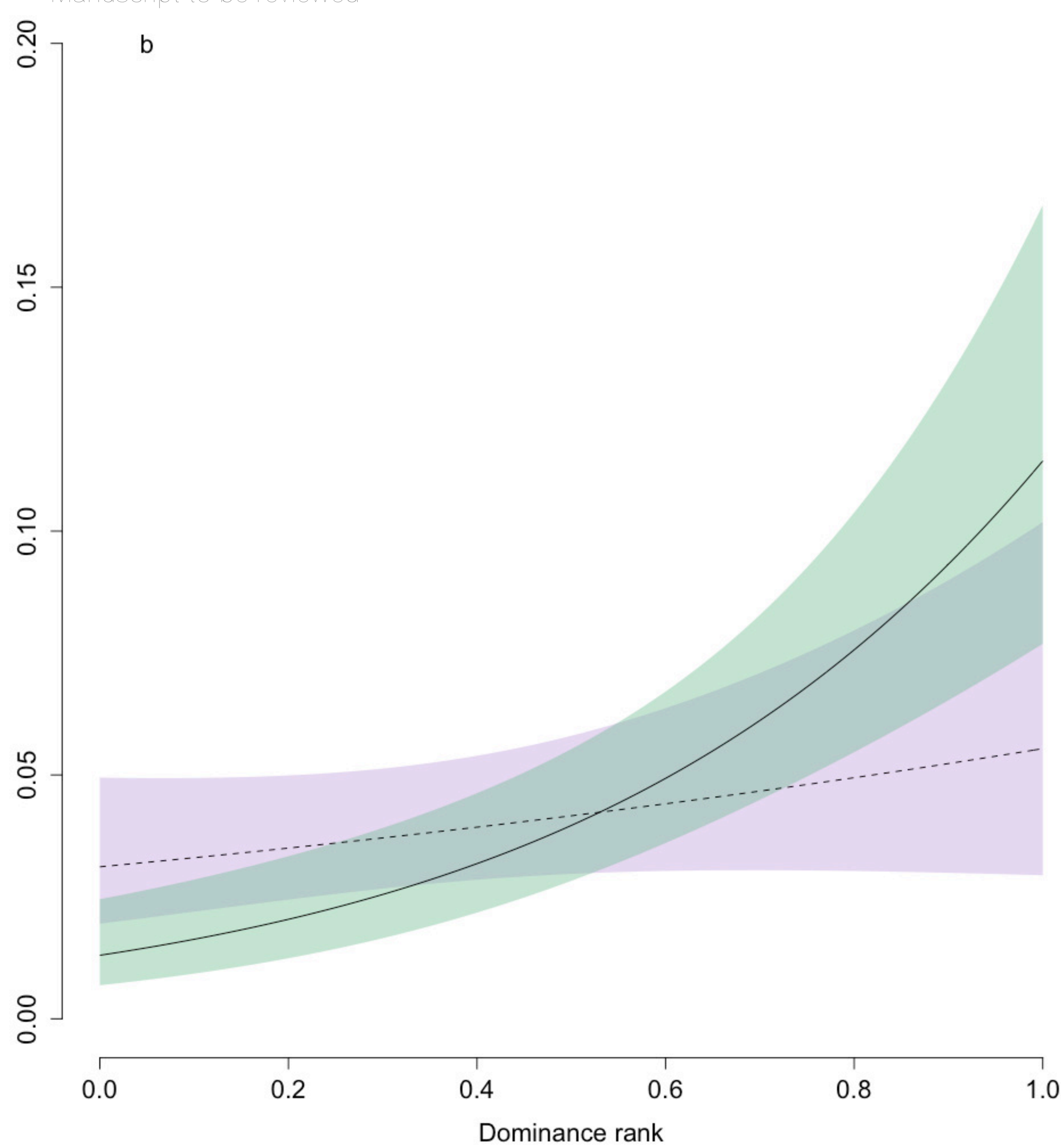




\section{Figure 2 (on next page)}

Comparison of observed dominance asymmetry during join events with simulated joining behaviour that is random with respect to rank difference.

Probability density distributions show expectations for the proportion of join events in which the joining individuals would be subordinate to the joined individual if their behaviour was random with respect to the rank of the joined individual. The distributions are for adults (a) and juveniles (b) at the patch level, and adults (c) and juveniles (d) at the sub-patch level, generated through 10,000 iterations of randomly selecting the individual to be joined at each join event. For each distribution, dotted vertical lines indicate the $95 \%$ tolerance intervals and solid vertical lines indicate our observed value. 


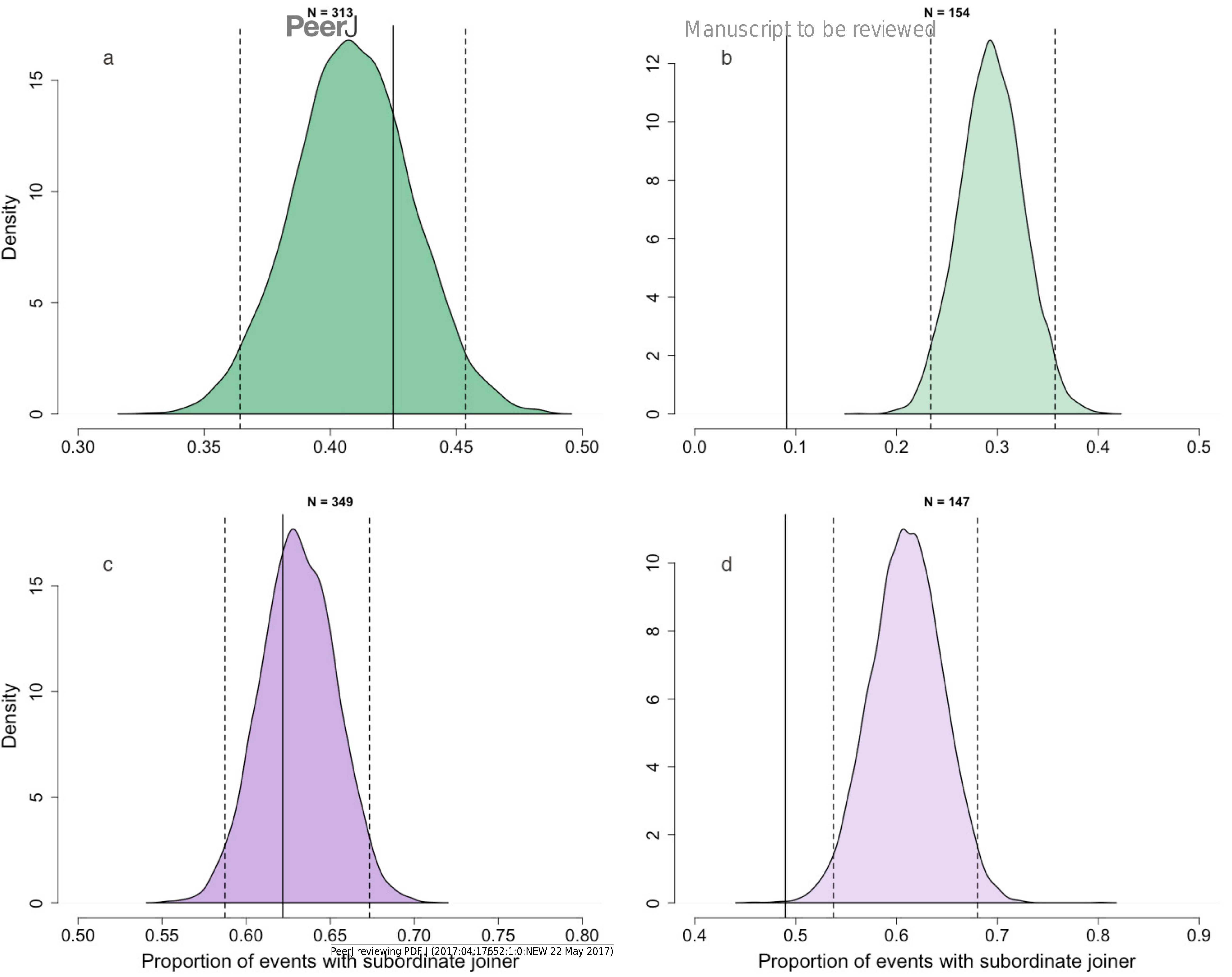

